\title{
Drug, Natural Product
}

National Cancer Institute

\section{Source}

National Cancer Institute. Drug, Natural Product. NCI Thesaurus. Code C1907.

Any drug derived from microorg anisms, plants, or animals; also, synthesized versions of these substances or their derivatives. 